\title{
Synthesis, characterization and cytotoxicity of polyethylene glycol-encapsulated CdTe quantum dots
}

\author{
Magdy Ali ${ }^{1,2} \cdot$ Dina Zayed $^{3} \cdot$ W. Ramadan ${ }^{4} \cdot$ Ola A. Kamel ${ }^{5} \cdot$ Mona Shehab $^{1,6} \cdot$ Shaker Ebrahim $^{1}$
}

Received: 4 October 2018 / Accepted: 17 December 2018 / Published online: 1 January 2019

(c) The Author(s) 2019

\begin{abstract}
Semiconductor quantum dots (QDs) offer tremendous scope to be used in the field of energy, imaging, sensors and optoelectronic devices. However, several issues regarding their hydrophilicity, stability and cytotoxicity remain unresolved. One of the methods to render CdTe QDs water-soluble is to functionalize the surface with carboxylate groups by the use of heterobifunctional ligands such as 3-mercaptopropionic acid (MPA). Following this motif, we report the synthesis of CdTe QDs using the non-aqueous organometallic route, ligand exchange with MPA was performed to replace the initial surface passivation ligands trioctylphosphine oxide (TOPO) and hexadecylamine, and finally, the prepared MPA-CdTe QDs were encapsulated into the biocompatible polyethylene glycol. Our results show that pegylation of CdTe QDs provided minimal cytotoxicity against baby hamster kidney (BHK 21) cells even at high levels. The $\mathrm{IC}_{50}$ values of pegylated CdTe QDs were higher than the non-pegylated ones with a \% viability ranges from 89:90\% at a concentration range of 0.01:0.03 nM. Currently, available ligand exchange procedures lead to significant loss of quantum yields. In our study, the photoluminescence (PL) emission of the MPA capped CdTe QDs decreased gradually as the $\mathrm{pH}$ increased from 4 to 10 while after pegylation the prepared QDs exhibited significantly enhanced PL efficiency and storage stability.
\end{abstract}

Keywords Quantum dots $\cdot$ CdTe $\cdot$ Toxicity $\cdot$ Encapsulated quantum dots $\cdot$ PEG $\cdot$ Ligand exchange

\section{Introduction}

Quantum dots (QDs) have attracted attention in recent years due to their potential applications [1]. For instance, effective labeling of fluorescent QDs is crucial for extracellular and intracellular tracking of target molecules in their native

Magdy Ali

igsr.magdyali@alexu.edu.eg

Dina Zayed

dinagzayed@hotmail.com

W. Ramadan

wegdan.ramadan@gmail.com

Ola A. Kamel

ola.kamel@pua.edu.eg

Mona Shehab

mona_shehab@alexu.edu.eg

Shaker Ebrahim

shaker.ebrahim@alexu.edu.eg

1 Department of Materials Science, Institute of Graduate

Studies and Research, Alexandria University, 163 Horrya

Avenue, El-Shatby, P.O. Box 832, Alexandria, Egypt environment [2]. QDs functionalized with antibody are optimal for extracellular targeting of cell-surface membrane proteins (e.g., receptors) and subsequent targeted imaging [3]. The brightness of CdSe QDs fluorescence has made them the widely labeled nanoparticles for various biosensing (e.g.,

2 European Egyptian Pharmaceutical Industries (EEPI), Km 25, Alexandria Cairo Desert Road. El Ameriya, Alexandria, Egypt

3 Department of Industrial Pharmacy, Faculty of Pharmacy, Alexandria University, Alexandria 21521, Egypt

4 Physics Department, Faculty of Science, Alexandria University, Alexandria, Egypt

5 Department of Pharmaceutics and Pharmaceutical Technology, Faculty of Pharmacy and Drug Manufacturing, Pharos University, Alexandria, Egypt

6 General Bureau of Beheira Governorate, Damanhour, Beheira 22111, Egypt 
oligonucleotides, organic dyes) and single or multiplex labeling (e.g., antibodies, peptides) [4].

CdTe QDs has narrow emission band, broad absorption and high fluorescence quantum yields [5, 6]. Different methods have been explored for the synthesis of CdTe QDs via aqueous method at $100{ }^{\circ} \mathrm{C}$ [7] or a non-aqueous organometallic route at high temperature (e.g., $300{ }^{\circ} \mathrm{C}$ ) [8]. Up to now, the most successful method to prepare highly luminescent CdTe QDs is the organometallic synthetic route, which uses TOPO and HDA as surface ligands. However, these methods require high temperatures, and the resulting QDs are insoluble in water, which makes the final product incompatible with the biological systems. The hydrophobic QDs should be converted to hydrophilic QDs by surface exchange with hydrophilic ligands in aqueous solution [9]. More recently, QDs have been prepared in aqueous medium in a simpler route generating less toxic water-soluble biologically compatible nanocrystals $[10,11]$. Nevertheless, they suffer from certain limitations. First, they possess lower quantum yields compared to those prepared in organic media [12]. Second, they tend to aggregate with time due to the weakly bound thiol ligands. Finally, the water-soluble CdTe QDs can be oxidized due to liberate the toxic cadmium ions to the surrounding environment. In particular, the UV irradiation for bioimaging will accelerate the oxidation process [13]. Therefore, researchers have investigated the influence of hydrophilic surface ligands exchange method so as to enhance hydrophilicity and improve the optical properties to be used in biological applications [14].

Earlier studies showed that ligands play an important role during the formation of nanocrystals, exerting a strong effect on both the nucleation and growth stages. Hence, surface ligands can control the size, shape, growth kinetics, and optical properties of the QDs [15]. Moreover, it has been proposed that QDs toxicity could be correlated to the nature of surface ligands rather than its type and so it may confer protection against the toxicity induced by heavy metals [16]. The most often used surface ligands are thioglycolic acid (TGA) or mercaptopropionic acid (MPA) [17]. There are three distinct regimes for surface modification and phasetransfer of CdTe QDs. A ligand exchange method in which the native hydrophobic ligands replaced by bifunctional ligands of surface anchoring thiol-containing molecules [18]. However, this method lowers the stability of QDs even with small particle size. The second method is depositing extra inorganic coatings such as $\mathrm{SiO}_{2}$ onto the surface of QDs. $\mathrm{SiO}_{2}$ coating enhances the stability of QDs and this approach leads to increase QDs size which is not preferred in biomedical applications. The last method is encapsulating of the QDs with biocompatible polymer such as PEG [19], dendron [20], and amphiphilic polymer [21].

Among various approaches, surface modification of QDs with polyethylene glycol (PEG) ligands has proved to be one of the most successful strategies [22]. (PEG) was considered to be the best biocompatible material which is well known to increase the circulation time by decreasing the clearance of QDs via the reticuloendothelial system (RES). They have high resistance to protein adsorption and therefore reduce nonspecific QDs-cell membrane interactions. Their hydrophilic and non-toxic nature also mitigates QDsinduced adverse effects by preventing chemical degradation of QDs and release of their metal ions to intracellular environments [23]. Encapsulated within the PEG polymer, the modified QDs could be highly biocompatible without any loss in fluorescence intensity of original QDs and could be useful for the discrimination of specific target by determining their luminescence emission. The main objective of this work is to address the problems of CdTe QDs concerning hydrophilicity, stability and cytotoxicity. CdTe QDs were synthesized using non-aqueous organometallic route followed by ligand exchange of HDA and TOP with MPA and then encapsulating the CdTe QDs in PEG. The cytotoxicity of CdTe QDs was determined by the 3-(4,5-dimethylthiazol2-yl)-2,5-diphenyltetrazolium bromide (MTT) assessment against baby hamster kidney (BHK 21) cells.

\section{Experimental}

\section{Chemicals}

All chemicals were of analytical grades and have been used without any further purification. MPA and hexane were supplied by Across Company; 3-(4,5-dimethylthiazol-2-yl)2,5-diphenyltetrazolium bromide (MTT), TOP (99.9\%), Te powder (99.99\%), hexadecylamine (HAD) and dimethyl sulfoxide (DMSO), and PEG 600 were purchased from SigmaAldrich Company (St. Louis, USA). Cadmium acetate (98\%) was obtained from Wako Chemical Reagent Company (California, USA). The $\mathrm{pH}$ was adjusted with $\mathrm{NaOH}$ or $\mathrm{HCl}$ that were obtained from Beijing Chemical Reagent Company (Beijing, China). Baby hamster kidney cells were purchased from the Medical Technology Center for Research and Services. Dulbecco's modified Eagle's medium (DMEM, Gibco, NY, USA) was supplied by Gibco (Invitrogen, USA) supplemented with $10 \%$ fetal bovine serum (FBS, Hyclone) and $1 \%$ penicillin/streptomycin (Hyclone).

\section{Synthesis of CdTe QDs}

CdTe QDs were prepared in non-aqueous solution according to our previously published method [24]. The procedure of synthesis of CdTe QDs using organometallic route is schematically described in Fig. 1. Briefly, in a three-necked round flask, a mixture of $\left(\mathrm{CH}_{3} \mathrm{COO}\right)_{2} \mathrm{Cd}(0.28 \mathrm{mmol})$, TOP $(3 \mathrm{~mL})$ and hexadecylamine (HDA, $2 \mathrm{~g}$ ) was refluxed at 


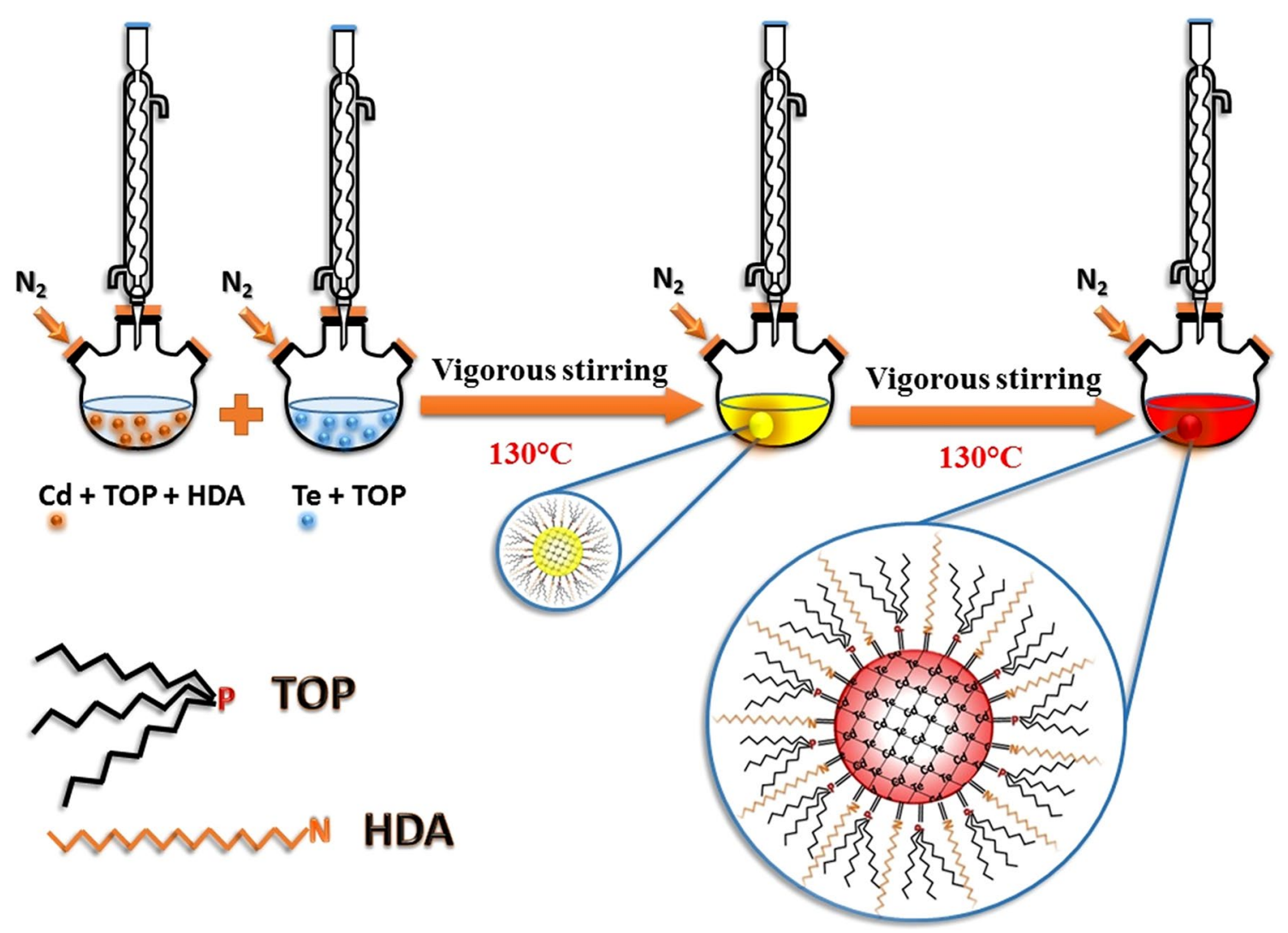

Fig. 1 Schematic illustration of synthesis of CdTe QDs through organometallic route

$130{ }^{\circ} \mathrm{C}$ under nitrogen atmosphere. Meanwhile, The TOP-Te solution was prepared by dissolving Te powder $(0.24 \mathrm{mmol})$ in $2 \mathrm{~mL}$ TOP. The mixture is then vigorously stirred at $100{ }^{\circ} \mathrm{C}$ under nitrogen atmosphere until the yellowish green color of Top-Te solution appears. TOP-Te solution was added to the cadmium solution. The growth and nucleation of CdTe QDs was developed at fixed temperature of $130^{\circ} \mathrm{C}$.

\section{Ligand exchange with MPA}

To exchange the hydrophobic capping agent of the CdTe QDs, CdTe QDs were diluted in hexane $(10 \mathrm{mg} / \mathrm{mL})$ and mixed with equal amounts of MPA for $2 \mathrm{~h}$ under smooth continuous stirring. Then, $5 \mathrm{~mL}$ of deionized water was added to the solution. After $30 \mathrm{~min}$ of continuous stirring, a clear colorless aqueous layer is formed above the clear hexane solution then the organic phase was removed. A clear aqueous solution of MPA capped CdTe QDs was obtained as shown in Fig. 2.

\section{Encapsulation of CdTe QDs}

A direct one-step protocol was used to encapsulate CdTe QDs with PEG-600. Briefly, MPA-capped CdTe QDs exchanged solution was added very slowly to an equal amount of PEG-600 under smooth stirring at $40{ }^{\circ} \mathrm{C}$ for $1 \mathrm{~h}$. The preparation step is illustrated in Fig. 2 yielding a clear water-soluble non-immunogenic PEG-600 encapsulated CdTe QDs solution.

\section{Characterization}

The structural property was studied by X-ray 7000 diffraction (Shimadzu-Japan) using copper characteristic wavelength with $1.54 \mathrm{~A}^{\circ}$. The $2 \theta$ angles of the diffractometer were collected from $2^{\circ}$ to $70^{\circ}$ at a scan rate of $10^{\circ} / \mathrm{min}$. The morphological property was investigated using Jeol JEM2100 transmission electron microscope (Tokyo, Japan) at an accelerating voltage of $200 \mathrm{kV}$. Samples for HRTEM were prepared by dispersing dried powder of CdTe QDs in ethanol and allowing a drop of this ethanol solution to dry on a 3-mm-diameter carbon-coated fine copper grid while the excess solution was wicked away by a filter paper. The grid was subsequently dried in air before the measuring step. The fluorescence intensity and the emission spectra were recorded with the excitation wavelength fixed at $450 \mathrm{~nm}$ using an LS 55 fluorescence spectrophotometer (Perkin Elmer, USA). The excitation slit and the emission slit were 
Fig. 2 Schematic illustration of ligand exchange mechanism and polymer encapsulation strategy. The hydrophobic CdTe QDs ligands on the surface of QDs were left intact (a) and a hydrophilic MPA was used to transfer the QDs in a water phase (b)

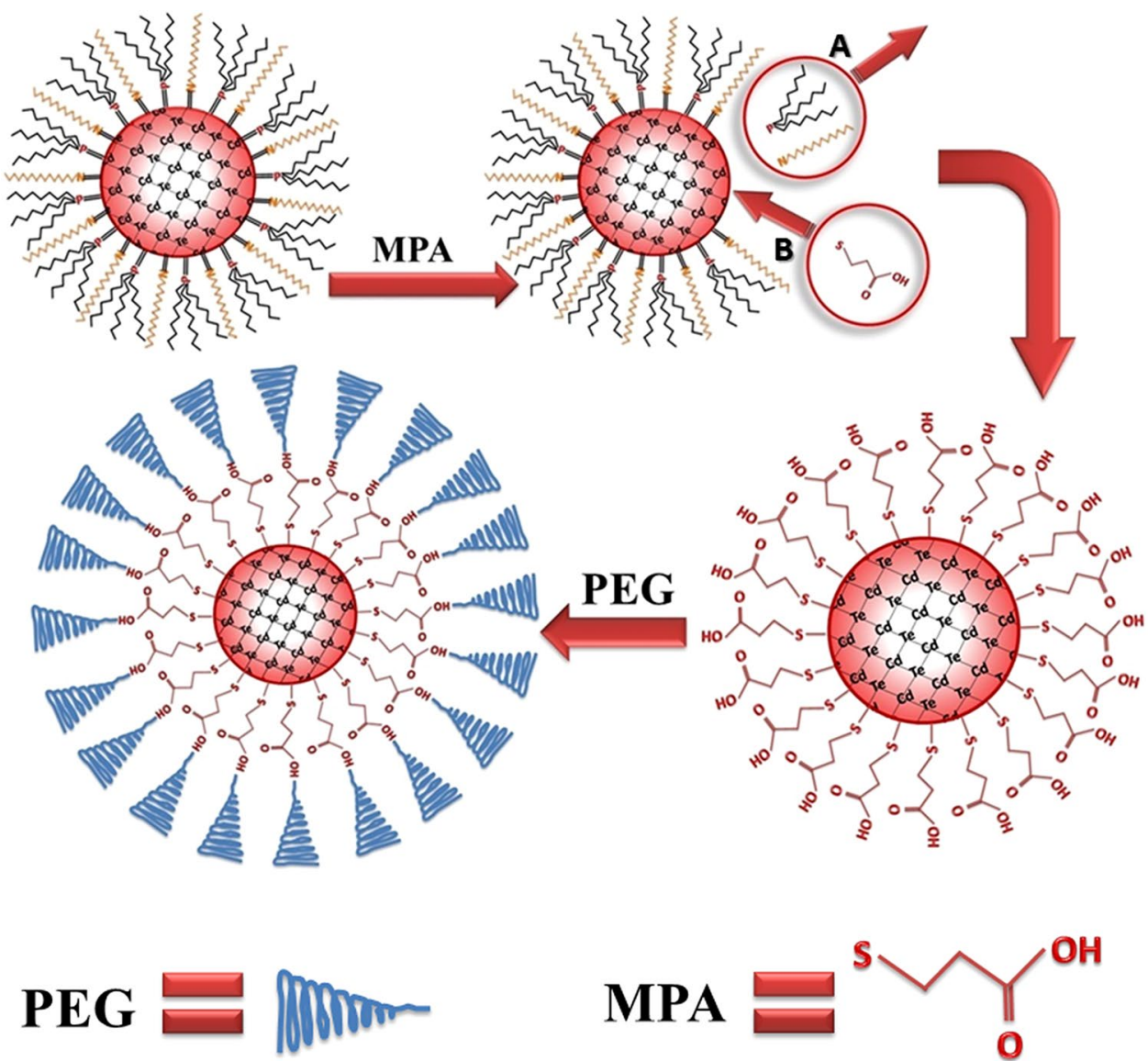

maintained at $5.0 \mathrm{~nm}$ and $10.0 \mathrm{~nm}$, respectively. The scan rate of the monochromators was maintained at $2400 \mathrm{~nm} / \mathrm{min}$. The absorption spectra of CdTe QDs were obtained using UV-visible spectrometer (Thermoevolution 2000, Thermo scientific, USA).

\section{Cytotoxicity of CdTe QDs and cell viability assay}

Cytotoxicity of different concentrations of CdTe QDs was determined by 3-(4,5-dimethylthiazol-2-yl)-2,5-diphenyltetrazolium bromide (MTT) assessment, a colorimetric laboratory test to monitor color change in living organisms due to the activity of certain mitochondrial enzymes that cause reduction of yellow colored MTT to the purple colored formazan. The change in the intensity of purple color was determined by the absorption spectrum at $550 \mathrm{~nm}$. Comparing cells injected with MPA-CdTe QDs and PEG encapsulated MPA-CdTe QDs gives us information about how the metabolic activity of cells as a result of any change in the mitochondrial activity [25].

The cell culture experiments were carried out on baby hamster kidney (BHK 21) cells. Cells were maintained in Dulbecco's modified Eagle's medium (DMEM) containing $10 \%$ fetal bovine serum (FBS) (BIO-IND), $50 \mu \mathrm{g} / \mathrm{mL}$ gentamicin sulfate, incubated at $37{ }^{\circ} \mathrm{C}$ in dark place with $5 \% \mathrm{CO}_{2}$ and $95 \%$ humidification. $95 \mu \mathrm{L}$ of cell suspension was incubated into 96-well microculture plates at a density of $1 \times 10^{4}$ cells per well in culture media containing FBS and gentamicin sulfate and allowed to adhere to the plate overnight. These cells were divided into three groups MPA-CdTe QDs, PEG encapsulated MPA-CdTe QDs and the control group [26]. The cytotoxicity of MPA-CdTe QDs and PEG encapsulated MPA-CdTe QDs was determined by the MTT assay method. Therefore, the medium was replaced by fresh medium containing different concentrations of MPA-CdTe QDs and PEG encapsulated MPA-CdTe QDs and incubated for another $24 \mathrm{~h}$. After that, the medium of the cells was separated and wells were thoroughly washed by pre-warmed phosphate-buffered saline (PBS) to take off any residual of the CdTe QDs. MTT stock solution $(5 \mathrm{mg} / \mathrm{mL})$ was diluted at 1:10 ratio into complete culture media, $100 \mu \mathrm{L}$ of MTT dilution was added into each well and incubated. After 3-5 h, medium was carefully removed by centrifugation at $2000 \mathrm{rpm}$ for $10 \mathrm{~min}$ to prevent any disturbance of the precipitated formazan crystals at the well bottom. Crystals were dissolved with $100 \mu \mathrm{L}$ DMSO. The absorbance was determined at $540 \mathrm{~nm}$. Results were represented as percentage viability and calculated by the following formula [27]: 
$\%$ Viability $=\left(\frac{\mathrm{OD}_{\mathrm{s}}-\mathrm{OD}_{\mathrm{b}}}{\mathrm{OD}_{\mathrm{c}}-\mathrm{OD}_{\mathrm{b}}}\right) \times 100$,

where $\mathrm{OD}_{\mathrm{b}}$ indicated the optical density of blank, $\mathrm{OD}_{\mathrm{s}}$ indicated the optical density of sample and $\mathrm{OD}_{\mathrm{c}}$ indicated the optical density of control.

The viability of BHK21 cells is measured for three different concentrations 0.0134: $0.213 \mathrm{nM}$ after $24 \mathrm{~h}$ of incubation with the MPA capped CdTe QDs and encapsulated PEG(MPA-CdTe) QDs. Thus, at each CdTe QDs concentration, three viability measurements are taken and the results are expressed as the mean of three independent experiments. To eliminate the background fluorescence, we included an untreated cell (maximum viability) control in the cytotoxicity assessment. The maximum viability control is established
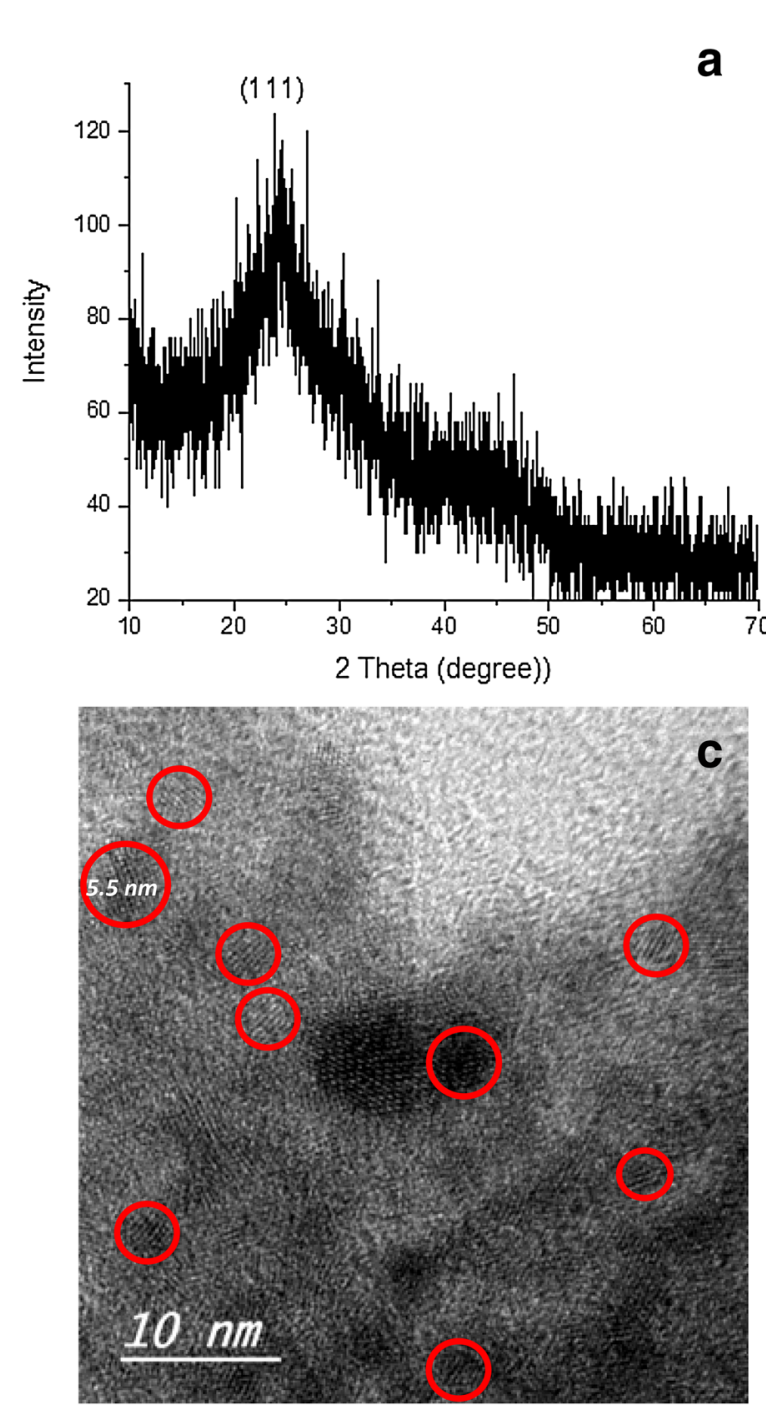

by adding the vehicle only (used to deliver the test compound to test wells).

\section{Results and discussion}

\section{Structural and morphological properties of CdTe QDs}

The XRD pattern of the CdTe QDs is shown in Fig. 3a. The position of the diffraction peaks was matched with that of the original cubic of the bulk CdTe in zinc blend phase [28]. Figure 3a depicted a broad peak typical for that of the bulk CdTe. Three diffractive peaks were noted and matched well with the (111), (220) and (311) reflections of cubic zinc blend CdTe [29]. The SAED pattern is shown in Fig. 3b. The diffuse rings are broad in the
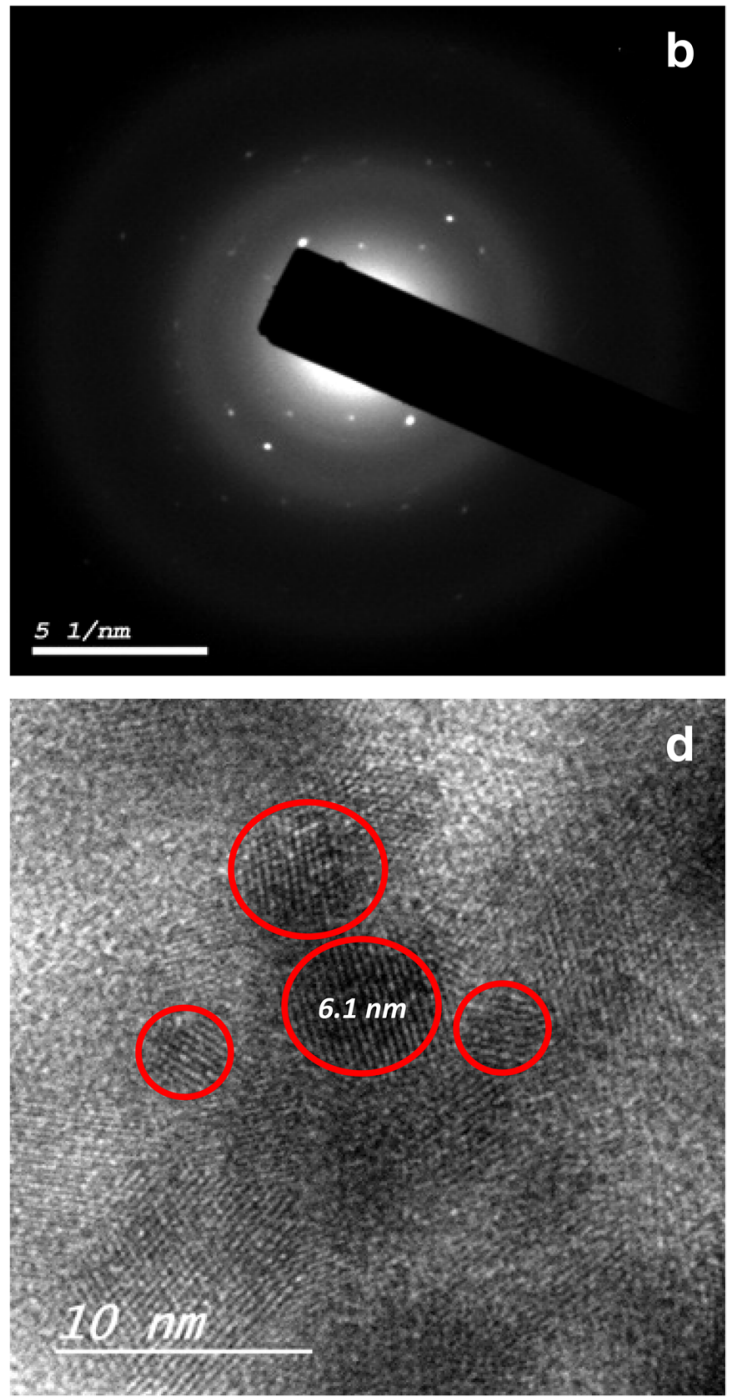

Fig. 3 XRD pattern of hydrophobic CdTe QDs (a), SAED (b), HRTEM images (c), and after 1 year on the shelf in solid form (d) 
electron diffraction pattern due to the small size of the QDs. The second ring in the SAED pattern in Fig. $3 b$ corresponds to a lattice spacing equal to about $0.26 \mathrm{~nm}$, which is indexed as the (102) plane [30]. The stability of the CdTe QDs prepared in non-aqueous phase has been conducted by comparing the newborn CdTe QDs with the CdTe QDs that has been stored for 1 year in a solid form using HRTEM images as indicated in Figs. $3 \mathrm{c}$ and $2 \mathrm{~d}$; the size of the CdTe QDs increased during the 1-year shelf life monitoring from $4-5.5 \mathrm{~nm}$ to $6-7.5 \mathrm{~nm}$. The increase in the size of the CdTe QDs is due to the aggregation of nanocrystals. As shown from these HRTEM images, the CdTe QDs have no significant change in their morphological and crystal structures. It can be concluded that the prepared CdTe QDs is stable in the shelf life up to 1 year with proper storage in the solid form.

Figure 4 shows the EDX spectrum of CdTe QD. Cd and Te peaks are observed at 3.13 , and $3.77 \mathrm{keV}$, respectively. These values are consistent with the values reported in the literature at 3.15 and $3.75 \mathrm{keV}$ for $\mathrm{Cd}$ and $\mathrm{Te}$, respectively [31]. Therefore, EDX results confirm the presence of $\mathrm{Cd}$ and Te which further indicates that CdTe QDs are prepared with Cd:Te ratio of about 1:1.2.

\section{Optical properties of CdTe QDs}

\section{Synthesis of CdTe QDs}

The $\left(\mathrm{CH}_{3} \mathrm{COO}\right)_{2} \mathrm{Cd}$ possess a poor solubility in TOP at room temperature and this in turn facilitates the controlled growth of CdTe QDs upon monitoring the temperature [30]. At $130{ }^{\circ} \mathrm{C}$, a dramatic change in color with time is observed. The synthesis procedure was found to be heterogeneous. Unlike homogenous reactions in which the growth of QDs occurs after nucleation at high temperatures at constant rate and hence the size of QDs can be controlled, in heterogeneous reactions the size cannot be controlled [32]. Aliquots of the reaction solution was taken at different time intervals (10:85 $\mathrm{min})$ and the absorption spectra was taken to monitor the growth of the clusters and the stability of CdTe QDs with time. At short time, the nucleation process and seed formation of CdTe QDs take place. Hence, dispersed nanocrystal CdTe QDs are formed.

The CdTe QD size can be calculated from the following equation [33]:

$D=\left(9.8127 \times 10^{-7}\right) \lambda^{3}-\left(1.7147 \times 10^{-3}\right) \lambda^{2}+(1.00064) \lambda-194.84$,

where $D(\mathrm{~nm})$ is the size of CdTe QDs, and $\lambda(\mathrm{nm})$ is the wavelength of the absorption peak.

The absorbance value was used to calculate the concentration of CdTe QDs using Beer's Law [34]:

$A=\varepsilon C L$,
Fig. 4 EDX spectrum of CdTe QDs

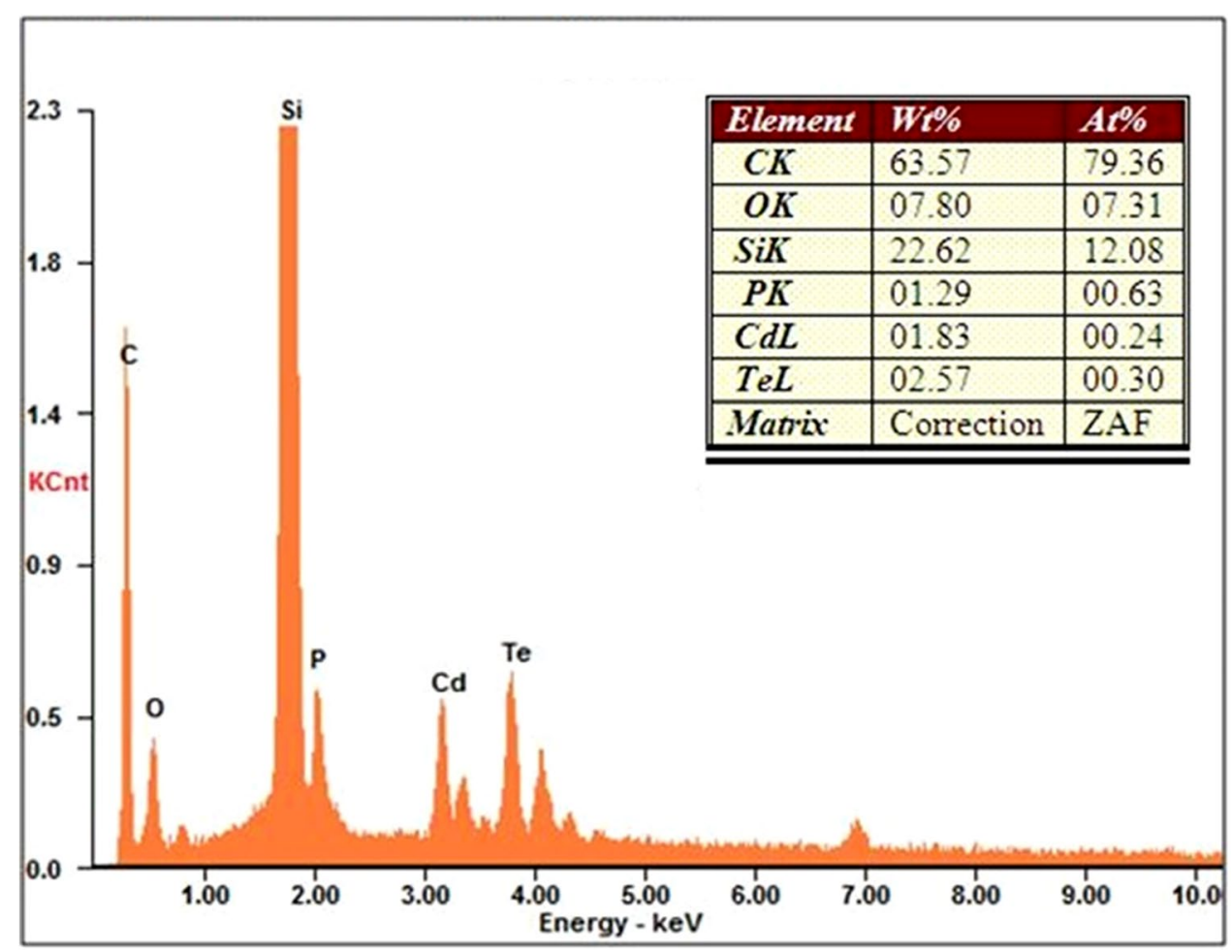


where $\varepsilon\left(\mathrm{L} \mathrm{mol}^{-1} \mathrm{~cm}^{-1}\right)$ is the extinction coefficient of CdTe QDs; $A$ is the absorbance at the peak, $l$ is the path length (cm), and $C$ is the molar concentration $\left(\mathrm{mol} \mathrm{L}^{-1}\right)$ of the CdTe QDs.

The extinction coefficient, $\varepsilon$, can be calculated using the following equation [35]:

$\varepsilon=10043(D)^{2.12}$,

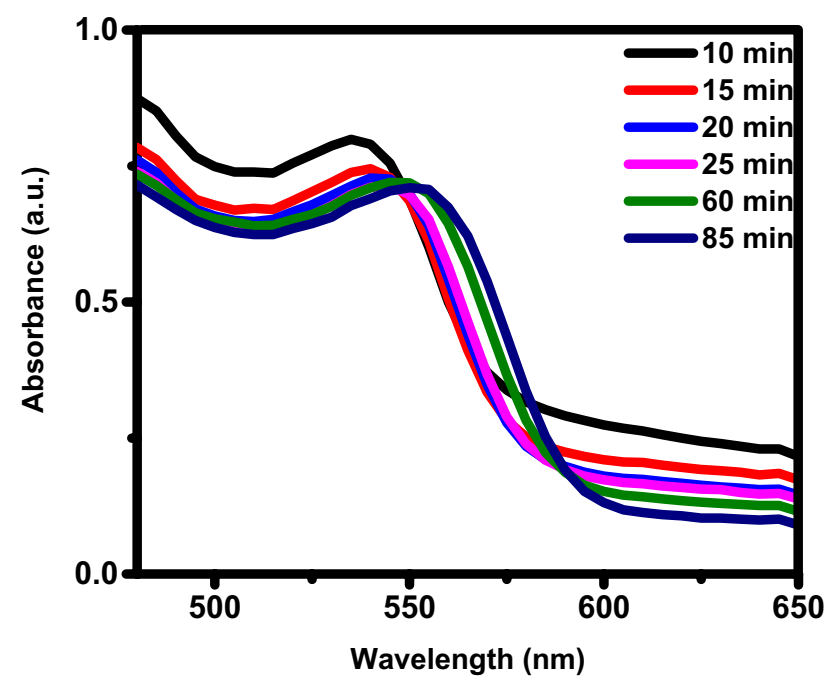

Fig. 5 Temporal evolution of UV-Vis absorption spectra of CdTe QDs during growth at 130 under nitrogen where $D(\mathrm{~nm})$ is the size of CdTe QDs. The $\varepsilon$ value is found to be $1.22 \times 10^{5} \mathrm{~cm}^{-1} \mathrm{~mol}^{-1} \mathrm{~L}$ and the molar concentration is $1.14 \times 10^{-6} \mathrm{~mol} / \mathrm{L}$.

Figure 5 showed the UV-visible spectra of the CdTe QDs prepared in non-aqueous phase at different time intervals. As the reaction time passes from 10:85 min, the absorption excitonic peak of CdTe QDs appears to red-shift due to the quantum confinement effect indicating the growth of QDs. With increase in size, the photon will be with comparatively lower energy and hence absorbs light at longer wavelengths. As seen in Fig. 5, the absorption peak of the CdTe QDs appeared at $535 \mathrm{~nm}$ and $554 \mathrm{~nm}$ after 10 and $85 \mathrm{~min}$, respectively. From Eq. 2, the size of the CdTe QDs reached $3.2 \mathrm{~nm}$ after $60 \mathrm{~min}$ of the reaction [36].

\section{Ligand exchange of CdTe QDs}

The ligand exchange process is carried out at room temperature under stirring and was allowed to continue for $2 \mathrm{~h}$. Figure 6a shows the progress of the ligand exchange reaction in which the hydrophobic CdTe QDs is separated out of the hexane phase and dissolved in water. No significance difference was observed in the PL spectrum of the newborn hydrophilic MPA capped CdTe QDs and the original hydrophobic QDs (Fig. 6b), while the PL intensity of the hydrophilic MPA capped CdTe QDs in water decreased from 450 a.u to 300 a.u at the same wavelength. A reduction in the PL is known when thiol-based ligands are assembled on the surface of the QDs [37]. This may be attributed to the short chain ligand of MPA in comparison with the TOP and
Fig. 6 CdTe QDs before and after the ligand exchange process (a) and PL spectra of CdTe QDs before (black) and after (red) the ligand exchange process (b)

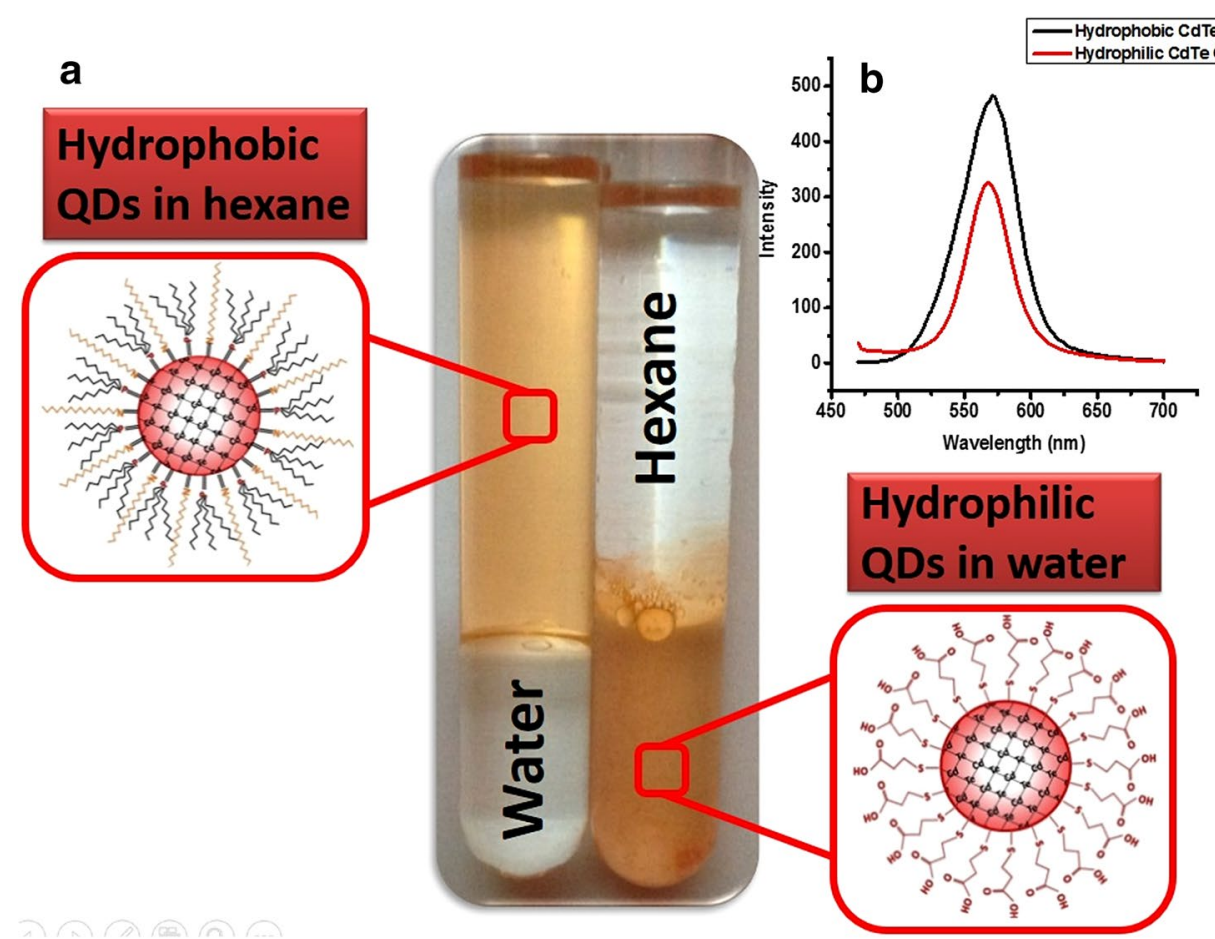


HDA chain which may in turn influence the agglomeration of the CdTe QDs [38].

\section{PEG encapsulated CdTe QDs}

Encapsulation of MPA-CdTe QDs within the PEG polymer was found to increase its water solubility and improve the QDs stability [39]. Being biocompatible, they possess the potential to detect and image different biomolecules. The emission spectra of PEG encapsulated MPA-CdTe QDs and the original hydrophobic QDs were recorded with the excitation wavelength fixed at $450 \mathrm{~nm}$ (Fig. 7a). The original hydrophobic CdTe QDs showed a characteristic symmetric emission peak at $571 \mathrm{~nm}$. A slight blue shift in the emission peak of the hydrophobic CdTe QDs was observed after encapsulation within PEG polymer, where the fluorescence peak shifted towards shorter wavelength from 571 to $556 \mathrm{~nm}$. A reduction of the PL was observed in the PEG encapsulated MPA capped CdTe QDs compared to the
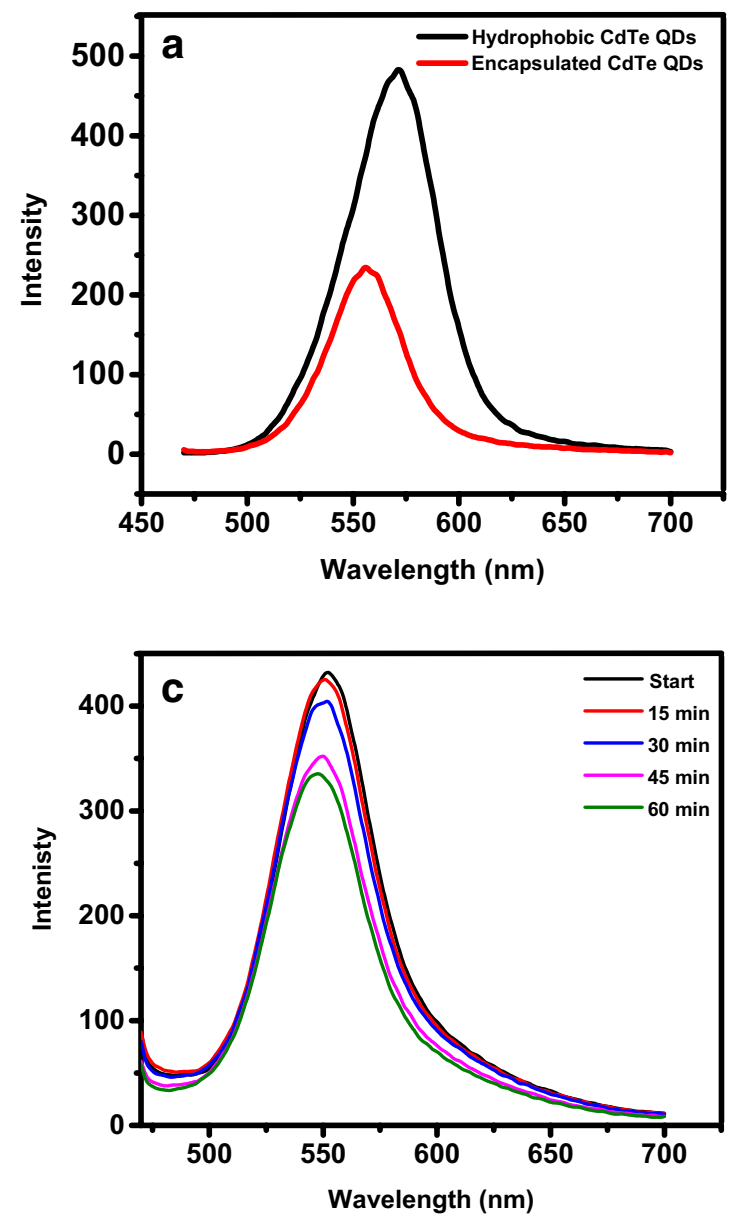

original hydrophobic QDs. This may be explained as a result of particle agglomeration during encapsulation process [40].

The stability and optical properties of the PEG encapsulated MPA-CdTe QDs and water-soluble MPA-QDs were examined after 3 weeks of storage in dark place and then compared to observe the effect of PEG encapsulation on CdTe QDs stability. The results show that the PEG encapsulated MPA-CdTe QDs remained stable with a slight decrease in the PL intensity from 450 a.u. to 100 a.u. when compared with the PL intensity of the freshly prepared QDs. On the other hand, a dramatic decline in the PL intensity and stability of the MPA capped CdTe QDs was observed after 3 weeks of storage (Fig. 7b). Moreover, the orange color of MPA-CdTe QDs disappeared on the third day of storage, while PEG encapsulated MPA-CdTe QDs remained stable without any color change. These results indicate that the encapsulation process of CdTe QDs within PEG polymer enhances QDs stability and photoluminescence properties. Figure 7c shows the temporal changes of the PL intensity of MPA-CdTe QDs under continuous UV irradiation. A slight
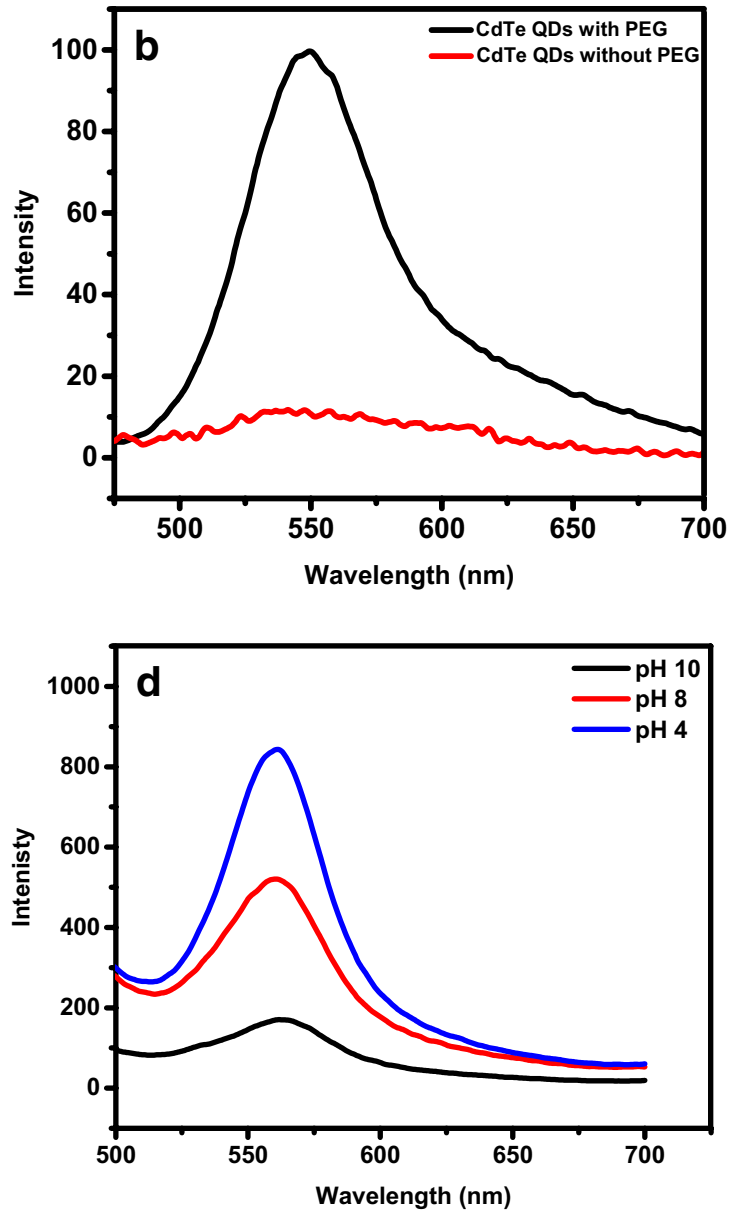

Fig. 7 PL spectra of hydrophobic CdTe and MPA capped CdTe QDs (a), CdTe QDs with and without encapsulation after 3 weeks (b), effect of continuous irradiation on MPA capped CdTe QD (c) and effect of pH (d) 
decrease in the PL intensity occurred during the period of measurement $(0: 60 \mathrm{~min})$. A makeable decline in the PL intensity was observed losing about 1.6, 6.5 and $19 \%$ of its initial reading values after 15,30 and 45 min, respectively, and then no further decrease in the PL intensity was observed.

\section{Effect of pH on PL spectra of CdTe QDs}

To understand the effect of aquatic conditions on MPA-CdTe QDs, we investigated the PL intensity of MPA-CdTe QDs under different $\mathrm{pH}$ values. Figure $7 \mathrm{~d}$ shows that the PL intensity of MPA-CdTe QDs is pH dependant and is enhanced upon decreasing the $\mathrm{pH}$ values. It is demonstrated that $\mathrm{Cd}^{2+}$ ions and MPA form polynuclear complexes in water and the formation step depends mainly on the $\mathrm{pH}$ value [41]. When the $\mathrm{pH}$ values decrease, free thiol groups and $\mathrm{Cd}^{2+}$ ions are released from the Cd-MPA complexes so more trap sites on the CdTe QDs surface are removed and this dramatically improves the fluorescence intensity [42]. In our study, the PL intensity of the MPA-CdTe QDs decreased gradually upon increasing the $\mathrm{pH}$ from 4.0 to 10.0 , providing an evidence that our prepared MPA-CdTe QDs would favor the acidic environment. This is in agreement with Zhengtao et al. who studied the $\mathrm{pH}$ effect on thioglycolic acid capped CdTe QDs [43].

\section{Cytotoxicity assessment of the CdTe QDs}

The rapid emergence of QDs as highly efficient biological imaging agents brought along safety concerns related to their metal-containing compositions. Herein, we aim to address the subsequent effect of PEG molecules encapsulating MPACdTe QDs on QDs-induced toxicity.

Cytotoxicity profile and cell viability assay with MPACdTe QDs and PEG encapsulated MPA-CdTe QDs are carried out using BHK-21 cells as shown in Fig. 8 grown on 96-well plate using MTT test with purple colored formazan. The QDs of $100 \mu \mathrm{L}$ and $10 \mu \mathrm{L}$ of the sample prepared as such were added to the cells. The results in Fig. 8 show that cell viability is decreased as a function of concentration. MTT assay is observed under different concentrations after $24 \mathrm{~h}$ incubation with the QDs.

MPA-CdTe QDs were observed to be more cytotoxic than PEG encapsulated QDs. PEG encapsulated MPACdTe QDs demonstrated very little toxicity to BHK 21 cell line (viability was $89: 90 \%$ at a concentration range 0.01:0.03 nM). On the other hand, the viability of BHK 21 shows a rapid decrease in case of the MPA capped CdTe QDs treated cells indicated by the dramatic reduction of $\%$ viability from 89 to $70 \%$ at the same narrow concentration range. At a concentration of $0.2 \mathrm{nM}$, PEG encapsulate

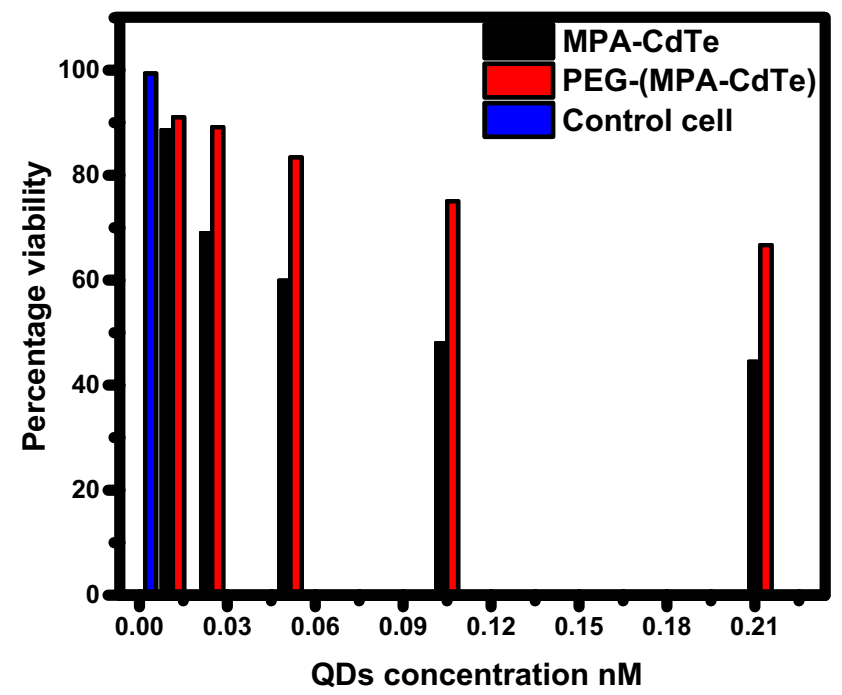

Fig. 8 Viability BHK 21 cells of MPA capped CdTe QDs and encapsulated PEG-MPA capped CdTe QDs versus QDs concentration using standard MTT colorimetric assays

MPA- CdTe QDs showed more than 65\% viability compared to the bare MPA-CdTe QDs which showed only $45 \%$ cell viability. The $50 \%$ cell viability (IC50) was determined to be less than $0.12 \mathrm{nM}$ for bare MPA-CdTe QDs. Comparatively, PEG encapsulated MPA-CdTe QDs were found to be less toxic as the IC50 was greatly improved to above $0.21 \mathrm{nM}$. It was found that QDs-induced toxicity is a function of MPA and PEG concentration [44]. These results suggested that embedding QDs in PEG increased their biocompatibility as is evident from the increased viability of exposed cells when compared to bare MPA-CdTe QDs. Similarly, Ulusoy et al. studied the cytotoxicity of MPA-functionalized CdTe/CdS/ZnS QDs and PEGylated $\mathrm{CdTe} / \mathrm{CdS} / \mathrm{ZnS}$ Qdots on A549 human lung adenocarcinoma cell lines and found that the $\mathrm{IC}_{50}$ value of pegylated QDs was $282.9 \pm 26.4 \mu \mathrm{g} / \mathrm{mL}$ in comparison to $21.4 \mu \mathrm{g} /$ $\mathrm{mL}$ for the non-pegylated ones [45]. On the basis of these observations, we can clearly state that the addition of PEG molecules to QDs surfaces minimized the negative effects of QDs by improving their colloidal stability and acted as a protective shield leading to reduction in QDs cytotoxicity.

\section{Conclusions}

In conclusion, we synthesized non-aqueous CdTe QDs followed by a ligand exchange of TOP and HDA with MPA forming the water-soluble MPA-CdTe QDs. To further 
decrease the QDs toxicity, the water-soluble MPA-CdTe QDs were encapsulated into PEG molecules. The PEG encapsulated CdTe QDs cytotoxicity was studied on BHK 21 cell line and compared to the non-pegylated QDs and the results showed that cells treated with pegylated QDs demonstrated higher \% viability than those treated with the non-pegylated ones. This is open new avenues towards preparation of biocompatible non-toxic QDs to be used for invivo and in vitro diagnostic and therapeutic applications.

Funding This work was not supported.

\section{Compliance with ethical standards}

Conflict of interest The authors declare that they have no conflict of interest.

Open Access This article is distributed under the terms of the Creative Commons Attribution 4.0 International License (http://creativeco mmons.org/licenses/by/4.0/), which permits unrestricted use, distribution, and reproduction in any medium, provided you give appropriate credit to the original author(s) and the source, provide a link to the Creative Commons license, and indicate if changes were made.

\section{References}

1. Shehab, M., Ebrahim, S., Soliman, M.: Graphene quantum dots prepared from glucose as optical sensor for glucose. J. Lumin. 184, 110-116 (2017)

2. Jain, S., Park, S.B., Pillai, S.R., Ryan, P.L., Willard, S.T., Feugang, J.M.: Applications of fluorescent quantum dots for reproductive medicine and disease detection. In: Gomes, A.C., Sarria, M.P. (eds.) Unraveling the Safety Profile of Nanoscale Particles and Materials-From Biomedical to Environmental Applications. InTech, London (2018)

3. Mashinchian, O., Johari-Ahar, M., Ghaemi, B., Rashidi, M., Barar, J., Omidi, Y.: Impacts of quantum dots in molecular detection and bioimaging of cancer. BioImpacts BI 4, 149 (2014)

4. Wang, Y., Hu, R., Lin, G., Roy, I., Yong, K.-T.: Functionalized quantum dots for biosensing and bioimaging and concerns on toxicity. ACS Appl. Mater. Interfaces 5, 2786-2799 (2013)

5. Labeb, M., Sakr, A.-H., Soliman, M., Abdel-Fettah, T.M., Ebrahim, S.: Effect of capping agent on selectivity and sensitivity of CdTe quantum dots optical sensor for detection of mercury ions. Opt. Mater. 79, 331-335 (2018)

6. Ebrahim, S., Labeb, M., Abdel-Fattah, T., Soliman, M.: CdTe quantum dots capped with different stabilizing agents for sensing of ochratoxin A. J. Lumin. 182, 154-159 (2017)

7. Ebrahim, S., Reda, M., Hussien, A., Zayed, D.: CdTe quantum dots as a novel biosensor for serratia marcescens and lipopolysaccharide. Spectrochim. Acta Part A Mol. Biomol. Spectrosc. 150, 212-219 (2015)

8. Pan, S., Ebrahim, S., Soliman, M., Qiao, Q.: Seed-mediated direct growth of CdSe nanoclusters on substrates. J. Nanopart. Res. 15, 1-7 (2013)

9. Mahmoudi, M., Meng, J., Xue, X., Liang, X.J., Rahman, M., Pfeiffer, C., Hartmann, R., Gil, P.R., Pelaz, B., Parak, W.J.:
Interaction of stable colloidal nanoparticles with cellular membranes. Biotechnol. Adv. 32, 679-692 (2014)

10. Priyam, A., Ghosh, S., Bhattacharya, S., Saha, A.: Supersaturation driven tailoring of photoluminescence efficiency and size distribution: a simplified aqueous approach for producing highquality, biocompatible quantum dots. J. Colloid Interface Sci. 333, 195-201 (2009)

11. Ali, M., El Nady, J., Ebrahim, S., Soliman, M.: Structural and optical properties of upconversion CuInS/ZnS quantum dots. Opt. Mater. 86, 545-549 (2018)

12. Cang, Y., Zhang, R., Shi, G., Fang, D., Guo, X.: Simple synthesis of high-quality CdTe QDs in spherical polyelectrolyte brushes with stable and reversible photoluminescence. Colloid Polym. Sci. 293, 3043-3047 (2015)

13. Derfus, A.M., Chan, W.C., Bhatia, S.N.: Probing the cytotoxicity of semiconductor quantum dots. Nano Lett. 4, 11-18 (2004)

14. Larson, D.R., Zipfel, W.R., Williams, R.M., Clark, S.W., Bruchez, M.P., Wise, F.W., Webb, W.W.: Water-soluble quantum dots for multiphoton fluorescence imaging in vivo. Science 300, 14341436 (2003)

15. Guerrini, L., Alvarez-Puebla, R.A., Pazos-Perez, N.: Surface modifications of nanoparticles for stability in biological fluids. Materials (Basel, Switzerland) 11, 1154 (2018)

16. Mehta, A., Sharma, S.N., Chawla, P., Chand, S.: Constraints in post-synthesis ligand exchange for hybrid organic (MEH-PPV)inorganic (CdSe) nanocomposites. Colloid Polym. Sci. 292, 301315 (2014)

17. Xu, S., Wang, C., Zhang, H., Wang, Z., Yang, B., Cui, Y.: pHsensitive photoluminescence for aqueous thiol-capped CdTe nanocrystals. Nanotechnology 22, 315703 (2011)

18. Liu, D., Snee, P.T.: Water-soluble semiconductor nanocrystals cap exchanged with metalated ligands. ACS Nano 5, 546-550 (2010)

19. Gerion, D., Pinaud, F., Williams, S.C., Parak, W.J., Zanchet, D., Weiss, S., Alivisatos, A.P.: Synthesis and properties of biocompatible water-soluble silica-coated $\mathrm{CdSe} / \mathrm{ZnS}$ semiconductor quantum dots. J. Phys. Chem. B 105, 8861-8871 (2001)

20. Jana, N.R., Earhart, C., Ying, J.Y.: Synthesis of water-soluble and functionalized nanoparticles by silica coating. Chem. Mater. 19, 5074-5082 (2007)

21. Zhou, C., Yuan, H., Shen, H., Guo, Y., Li, X., Liu, D., Xu, L., Ma, L., Li, L.S.: Synthesis of size-tunable photoluminescent aqueous $\mathrm{CdSe} / \mathrm{ZnS}$ microspheres via a phase transfer method with amphiphilic oligomer and their application for detection of HCG antigen. J. Mater. Chem. 21, 7393-7400 (2011)

22. Harris, J.M.: Introduction to biotechnical and biomedical applications of poly (ethylene glycol). In: Gomes, A.C., Sarria, M.P. (eds.) Poly (Ethylene Glycol) Chemistry, pp. 1-14. Springer, New York (1992)

23. Pelaz, B., del Pino, P., Maffre, P., Hartmann, R., Gallego, M., Rivera-Fernández, S., de la Fuente, J.M., Nienhaus, G.U., Parak, W.J.: Surface functionalization of nanoparticles with polyethylene glycol: effects on protein adsorption and cellular uptake. ACS Nano 9, 6996-7008 (2015)

24. Ebrahim, S., Ramadan, W., Ali, M.: Structural, optical and ferromagnetic properties of cobalt doped CdTe quantum dots. J. Mater. Sci. Mater. Electron. 27, 3826-3833 (2016)

25. Klein, R., Kellermeier, M., Touraud, D., Müller, E., Kunz, W.: Choline alkylsulfates-new promising green surfactants. J. Colloid Interface Sci. 392, 274-280 (2013)

26. Ciapetti, G., Cenni, E., Pratelli, L., Pizzoferrato, A.: In vitro evaluation of cell/biomaterial interaction by MTT assay. Biomaterials 14, 359-364 (1993)

27. Rodríguez-Lorenzo, L., García-Carrodeguas, R., Rodriguez, M., De Aza, S., Jiménez, J., López-Bravo, A., Fernandez, M., Román, J.S.: Synthesis, characterization, bioactivity and 
biocompatibility of nanostructured materials based on the wollastonite poly (ethylmethacrylate-co-vinylpyrrolidone) system. J. Biomed. Mater. Res. Part A 88, 53-64 (2009)

28. Talapin, D.V., Haubold, S., Rogach, A.L., Kornowski, A., Haase, M., Weller, H.: A novel organometallic synthesis of highly luminescent CdTe nanocrystals. J. Phys. Chem. B 105, 2260-2263 (2001)

29. Chao, M.-R., Chang, Y.-Z., Chen, J.-L.: Hydrophilic ionic liquid-passivated CdTe quantum dots for mercury iondetection. Biosens. Bioelectron. 42, 397-402 (2013)

30. Jose, R., Biju, V., Yamaoka, Y., Nagase, T., Makita, Y., Shinohara, Y., Baba, Y., Ishikawa, M.: Synthesis of CdTe quantum dots using a heterogeneous process at low temperature and their optical and structural properties. Appl. Phys. A 79, 1833-1838 (2004)

31. Haydous, F., Halaoui, L.: Quantum-confined CdTe films deposited by SILAR and their photoelectrochemical stability in the presence of Se2-as a hole scavenger. J. Phys. Chem. C 118, 18334-18342 (2014)

32. Peng, X., Wickham, J., Alivisatos, A.: Kinetics of II-VI and III-V colloidal semiconductor nanocrystal growth: "focusing" of size distributions. J. Am. Chem. Soc. 120, 5343-5344 (1998)

33. Wang, Y., Liu, S., Yang, K., Zhou, L.: One-pot synthesis of CdTe quantum dots using tellurium dioxide as a tellurium source in aqueous solution. Colloid Polym. Sci. 291, 1313-1318 (2013)

34. Maikala, R.V.: Modified Beer's Law-historical perspectives and relevance in near-infrared monitoring of optical properties of human tissue. Int. J. Ind. Ergon. 40, 125-134 (2010)

35. Yu, W.W., Qu, L., Guo, W., Peng, X.: Experimental determination of the extinction coefficient of $\mathrm{CdTe}, \mathrm{CdSe}$, and CdS nanocrystals. Chem. Mater. 15, 2854-2860 (2003)

36. Wang, Y., Wang, R., Liu, S., Yang, K., Zhou, L., Li, H.: Synthesis and characterization of CdTe quantum dots by one-step method. Bull. Chem. Soc. Ethiop. 27, 387-393 (2013)

37. Stewart, M.H., Susumu, K., Mei, B.C., Medintz, I.L., Delehanty, J.B., Blanco-Canosa, J.B., Dawson, P.E., Mattoussi, H.: Multidentate poly(ethylene glycol) ligands provide colloidal stability to semiconductor and metallic nanocrystals in extreme conditions. J. Am. Chem. Soc. 132, 9804-9813 (2010)

38. Sharma, H., Sharma, S.N., Singh, G., Shivaprasad, S.M.: Studies of optical and structural properties of $\mathrm{CdSe} /$ polymer nanocomposites: evidence of charge transfer and photostability. Colloid Polym. Sci. 285, 1213-1227 (2007)

39. Yu, W.W., Chang, E., Falkner, J.C., Zhang, J., Al-Somali, A.M., Sayes, C.M., Johns, J., Drezek, R., Colvin, V.L.: Forming biocompatible and nonaggregated nanocrystals in water using amphiphilic polymers. J. Am. Chem. Soc. 129, 2871-2879 (2007)

40. Wang, Y., Dave, R.N., Pfeffer, R.: Polymer coating/encapsulation of nanoparticles using a supercritical anti-solvent process. J. Supercrit. Fluids 28, 85-99 (2004)

41. Wang, Y., Liu, S.: One-pot synthesis of highly luminescent CdTe quantum dots using sodium tellurite as tellurium source in aqueous solution. J. Chil. Chem. Soc. 57, 1109-1112 (2012)

42. Lei, Y., Jiang, C., Liu, S., Miao, Y., Zou, B.: A clean route for preparation of CdTe nanocrystals and their conjugation with bacterium. J. Nanosci. Nanotechnol. 6, 3784-3788 (2006)

43. Deng, Z., Zhang, Y., Yue, J., Tang, F., Wei, Q.: Green and orange CdTe quantum dots as effective $\mathrm{pH}$-sensitive fluorescent probes for dual simultaneous and independent detection of viruses. J. Phys. Chem. B 111, 12024-12031 (2007)

44. Dubertret, B., Skourides, P., Norris, D.J., Noireaux, V., Brivanlou, A.H., Libchaber, A.: In vivo imaging of quantum dots encapsulated in phospholipid micelles. Science 298, 1759-1762 (2002)

45. Ulusoy, M., Jonczyk, R., Walter, J.-G., Springer, S., Lavrentieva, A., Stahl, F., Green, M., Scheper, T.: Aqueous synthesis of PEGylated quantum dots with increased colloidal stability and reduced cytotoxicity. Bioconjug. Chem. 27, 414-426 (2015)

Publisher's Note Springer Nature remains neutral with regard to jurisdictional claims in published maps and institutional affiliations. 